\title{
A Versatile 200-V Capacitor-Coupled Level Shifter for Fully Floating Multi-MHz Gate Drivers
}

\author{
Van Ha Nguyen ${ }^{\dagger}, \mathrm{Nam} \mathrm{Ly}^{\dagger}$, Abdul Hafiz Alameh ${ }^{\dagger}$, Yves Blaquière ${ }^{\dagger}$ and Glenn Cowan* \\ ${ }^{\dagger}$ Department of Electrical Engineering, École de technologie supérieure (ÉTS), University of Quebec, Montreal, Quebec, Canada \\ ${ }^{*}$ Department of Department of Electrical and Computer Engineering, Concordia University, Montreal, Quebec, Canada \\ E-mail: van-ha.nguyen.1@ens.etsmtl.ca
}

\begin{abstract}
This paper presents a novel level-shifter circuit for high-frequency high-voltage (HV) gate-drives. The proposed level shifter (LS) is designed based on a capacitivecoupler/current mirror/ latch structure which helps to extend operation voltage of a floating supply into the negative range, achieves sub-ns and constant delay, and consumes very low power from the floating supply. Additionally, common-mode noise cancellers based on a cross-current mirror and transmission gates are also presented to enhance the $\mathrm{dV} / \mathrm{dt}$ immunity of the LS against slewing of the floating ground. Implemented in $0.18 \mu \mathrm{m}$ HV BCD-on-SOI (bipolar-CMOSDMOS on silicon-on-isolator) process, the post-layout simulation of the proposed design shows a delay of $680 \mathrm{ps,}$ $200 \mathrm{~V} / \mathrm{ns}$ of $\mathrm{d} V_{\mathrm{SSF}} / \mathrm{dt}$ slew rate immunity, It dissipates no static power and only $8.1 \mathrm{pJ} /$ transition from the floating supply, improving FoM ${ }^{1}$ and $\mathrm{FoM}^{2}$ of the proposed LS by 3 times and 11.7 times compared to respective state-of-the-art works.
\end{abstract}

Keywords-Capacitive-coupled level shifter, BCD-on-SOI, floating level shifter, gate driver, GaN, level shifter, sub-ns delay.

\section{INTRODUCTION}

The level shifter (LS) circuit of the gate driver (GD) is an essential block that shifts the ground referenced pulse-width modulation (PWM) signal to different voltage domains. Fig. 1(a) shows a common design of GD used for half-bridge (HB) based DC-DC converters with the LS is highlighted [1]. The LS transfers a PWM signal from the low side to the high-side buffer for driving the high-side power switch. The four main challenges for the LS design in this context are:

(Ch1) Low propagation delay: the delay of the GD is mainly determined by the delay of the LS. For multi-MHz and/or high conversion ratio DC-DC converters, a LS with sub-ns delay can fully exploit the high switching speed of GaN FETs [2-3].

(Ch2) High $\mathrm{d} V_{\mathrm{SsF}} / \mathrm{d} t$ immunity: modern high-switching DC-DC converters use N-type MOS/GaN FETs as they offer low $R_{\mathrm{DS}, \mathrm{ON}} \times \mathrm{Q}_{\mathrm{g}}$. When driving the HS N-type FETs as shown in Fig. 1(a), the LS would suffer from high $\mathrm{d} V_{\mathrm{SW}} / \mathrm{d} t$ $\left(\mathrm{d} V_{\mathrm{SSF}} / \mathrm{d} t\right)$ that can erroneously flip the its output. For high-voltage (HV) applications using GaN FETs, $\mathrm{d} V_{\mathrm{SSF}} / \mathrm{d} t$ can be up to $100 \mathrm{~V} / \mathrm{ns}$; thus, a LS with $\mathrm{d} V_{\mathrm{SSF}} / \mathrm{d} t>100 \mathrm{~V} / \mathrm{ns}$ immunity is needed [3].

(Ch3) Low energy consumption: the LS consumes power from the floating supply (modeled as $C_{\mathrm{B}}$ ). The LS with high power consumption results in a high loss and thermal issues of the GD. Modern gate drivers prefer fully on-chip floating supplies whose power capability is limited. Therefore, the LS should consume as little power as possible [4].

(Ch4) Negative $V_{\mathrm{SSF}}$ tolerance: there are applications where $V_{\mathrm{SSF}}$ is negative. For example, during the dead-time $V_{\mathrm{SW}}=V_{\mathrm{SSF}}=-V_{\mathrm{D}}$ (forward voltage of body diode). In general, $V_{\mathrm{D}, \mathrm{MOSFET}}<1.5 \mathrm{~V}$ while $V_{\mathrm{D}, \mathrm{GaN}}$ can be up to $4.5 \mathrm{~V}$ [5]; thus, the remaining headroom $V_{\mathrm{DDF}}-V_{\mathrm{SSL}}$ is small such that it causes longer delay or even failure of the LS. The scenario becomes more critical if $V_{\mathrm{SSF}}$ is more negative with the effect of current sensing resistor $R_{\mathrm{S}}$ and/or parasitic $L_{\mathrm{par}}$. With new emerging DC-DC converters such as hybrid ones [6-8], $V_{\mathrm{SW}}$ can be in positive or negative domain as shown in Fig. 1(b). This extends the challenge (Ch4) such that the LS should work even with a large negative potential of the floating ground $V_{\mathrm{SSF}}$.

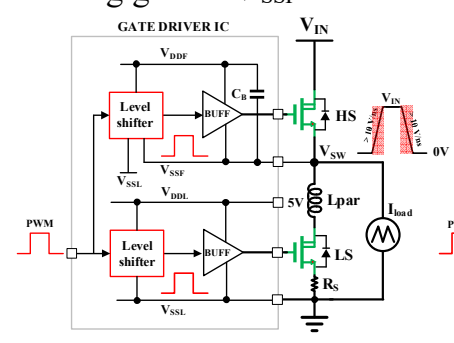

(a)

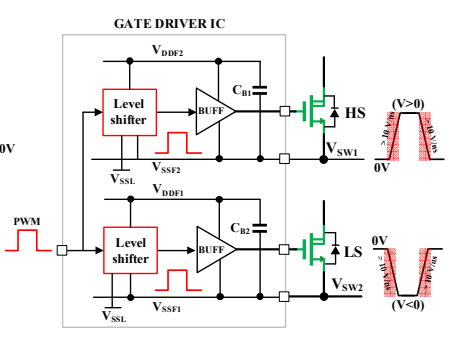

(b)
Fig. 1. High-voltage gate driver for: (a) HB-based DC-DC converters, (b) DC-DC converters with different floating sides.

Several level shifters have been proposed [9-17], which can be divided into two main categories: active-coupled LS (AC-LS) [9-15] and capacitive-coupled LS (CC-LS) [1617]. AC-LSs presented in [9-11] achieve a delay of $3 \mathrm{~ns}$, $2.03 \mathrm{~ns}$ and $1.3 \mathrm{~ns}$, respectively. However, these works cannot be used in systems where $V_{\mathrm{SSF}}$ slews fast and/or $V_{\mathrm{SSF}}$ is negative. AC-LSs with $\mathrm{d} V_{\mathrm{SSF}} / \mathrm{d} t$ slewing immunity are also reported [12-14]. The work in [13] presents a $\mathrm{d} V_{\mathrm{SSF}} / \mathrm{d} t$ immunity up to $120 \mathrm{~V} / \mathrm{ns}$ but has a high delay of $20 \mathrm{~ns}$. The work in [14] has only $0.37 \mathrm{~ns}$ delay but its $\mathrm{d} V_{\mathrm{SSF}} / \mathrm{d} t$ immunity is limited at $30 \mathrm{~V} / \mathrm{ns}$. In these works, negative $V_{\text {SSF }}$ tolerance is not reported. The recent work in [15] presents an excellent LS for SMPS applications with a $200 \mathrm{~V} / \mathrm{ns} \mathrm{d} V_{\mathrm{SSF}} / \mathrm{d} t$ immunity, $0.53 \mathrm{~ns}$ delay, but minimum $V_{\text {SSF }}$ is only $-1.5 \mathrm{~V}$. Though [12-15] can be applied to conventional HB-based DC-DC converters, even few negative volts of $V_{\mathrm{SSF}}$ would affect their reliable operation. These AC-LSs also cannot be used for GDs for emerging converters, as $V_{\mathrm{SSF}}$ is in negative domain [6-8]. Digital isolators are widely used as LSs to transmit the PWM signal, whether $V_{\text {SSF }}$ is negative or positive. However, this solution adds more complexity, cost, and size to the GD designs [18]. Furthermore, high-power consumption leads to a large $C_{\mathrm{B}}$ which is difficult to integrate $C_{\mathrm{B}}$ on-chip as the power consumption is high and scaled-up with $V_{\mathrm{DDF}}$ in these AC-LSs. Regarding CC-LS designs, reported works in $[4,16]$ also achieve sub-ns delays but are used for static

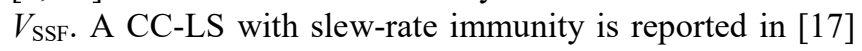


with $1.45 \mathrm{~ns}$ delay and consumes only $4.1 \mathrm{pJ} /$ transition, but $\mathrm{d} V_{\mathrm{SSF}} / \mathrm{d} t$ is limited to $6 \mathrm{~V} / \mathrm{ns}$. In short, the reported works [9-17] are not able to satisfy all four design challenges; thus, limiting their use to specific applications.

To tackle these issues, this paper presents a versatile LS for $\mathrm{HV}$ gate drivers that simultaneously achieves four design criteria (Ch1-Ch4). The proposed LS achieves 680 ps delay, $200 \mathrm{~V} / \mathrm{ns} \mathrm{d} V / \mathrm{d} t$ immunity, $8.1 \mathrm{pJ} /$ transition and can work under negative floating supply. Therefore, it can be applied to both conventional DC-DC converters and emerging ones. The remainder of the paper is organized as follows: Section II presents the proposed LS. The performance of the proposed LS is verified with intensive post-layout simulation results in Section III. Section IV concludes the paper.

\section{Proposed CAPACITIVE-Couple LEVEl ShIFTERS}

\section{A. Basic capacitive-coupled level shifter}

Fig. 2 shows the proposed basic CC-LS (BCC-LS) which is composed of three parts: (1) a pulse generator at low side, (2) two symmetrical current mirrors, a back-to-back inverterbased dynamic latch, and two buffer inverters at the floating side, (3) two coupling capacitors $C_{1}, C_{2}$ that connects the low side and the floating side. All devices are standard lowvoltage CMOS devices, except the HV coupling capacitors.

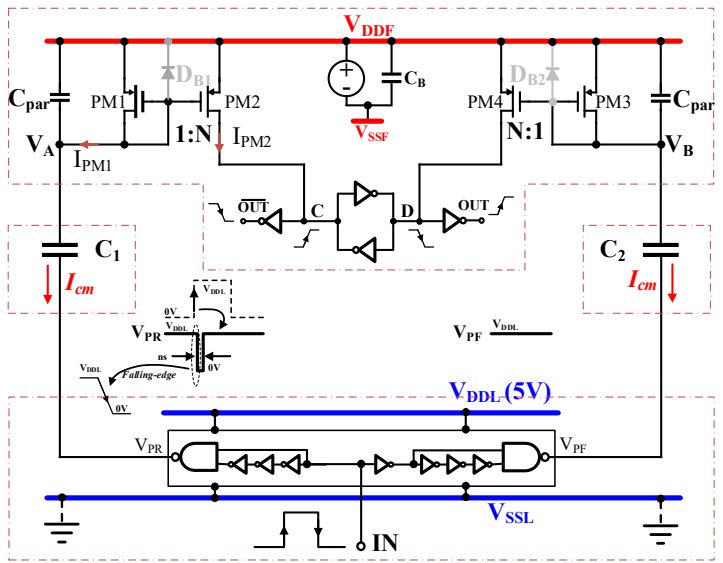

Fig. 2. The proposed basic capacitive-coupled level shifter with risingedge operation. (Red dashed boxes are deep N-wells for isolations).

The operation of the proposed BCC-LS is also shown in Fig. 2 (for the rising-edge operation) and its key timing waveform is shown in Fig. 3. On the rising transition of the input signal, a short pulse is generated at the output $V_{\mathrm{PR}}$ of the pulse generator while the output $V_{\mathrm{PF}}$ is maintained at $V_{\mathrm{DDL}}$. As $V_{\mathrm{PF}}$ does not change, the current mirrors PM3 and PM4 are inactive. Assume that $C_{1}, C_{2}$ is much higher than $C_{\mathrm{par}}\left(C_{\mathrm{par}}=C_{\mathrm{ds}, \mathrm{PM} 1}+C_{\mathrm{gs}, \mathrm{PM} 1,2}\right)$, a falling edge of the short pulse $V_{\mathrm{PR}}$ pulls the voltage $V_{\mathrm{A}}$ down from $V_{\mathrm{DDF}}$ to $V_{\mathrm{DDF}}-$ $V_{\mathrm{DDL}}$; thus, generating a current $I_{\mathrm{PM} 1}$ on the $C_{\mathrm{C} 1}-\mathrm{PM} 1$. This current is copied through the 1: $N$ current mirror PM1-PM2 to charge the node $\mathrm{C}$. As the triggering current $I_{\mathrm{PM} 2}$ is enough to pull up node $\mathrm{C}$ to exceed the threshold voltage of the latch, the positive feedback of the latch accelerates node $\mathrm{C}$ to $V_{\mathrm{DDF}}$ and node $\mathrm{D}$ to $V_{\mathrm{SSF}}$. Even when the triggering current reduces to zero, nodes $\mathrm{C}$ and $\mathrm{D}$ of the latch are held, ensuring the output is kept until the next change at the input IN. The inverter flips the voltage at node D so that the input and output signal are equal. In the same fashion, at the falling edge of the input signal, the right half is activated and a current $I_{\mathrm{PM} 4}$ is generated to change the state of the

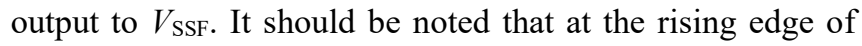
the short pulse at $V_{\mathrm{PR}}$ and $V_{\mathrm{PF}}$ of the pulse generator, capacitors $C_{1}$ and $C_{2}$ are discharged to the floating supply capacitor $C_{\mathrm{B}}$ through the body diode of PM1 and PM3, thus clamping $V_{\mathrm{A}}$ and $V_{\mathrm{B}}$ at a maximum level of $V_{\mathrm{DDF}}+V_{\mathrm{D}}$ where $V_{\mathrm{D}}$ is forward voltage of the body diode $\mathrm{D}_{\mathrm{B} 1}, \mathrm{D}_{\mathrm{B} 2}$.

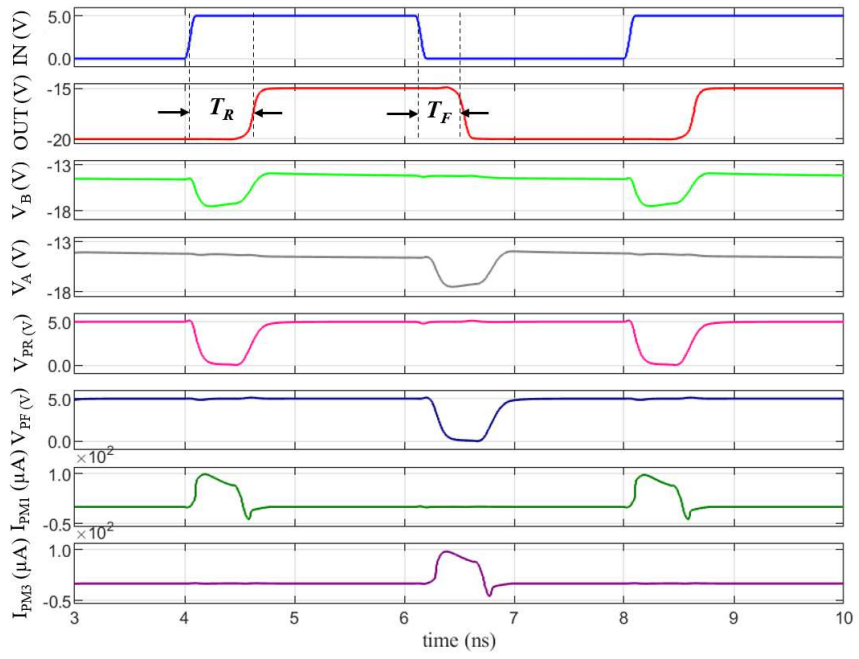

Fig. 3. Simulated waveform of the BCC-LS at $V_{\mathrm{SSF}}=-20 \mathrm{~V}$.

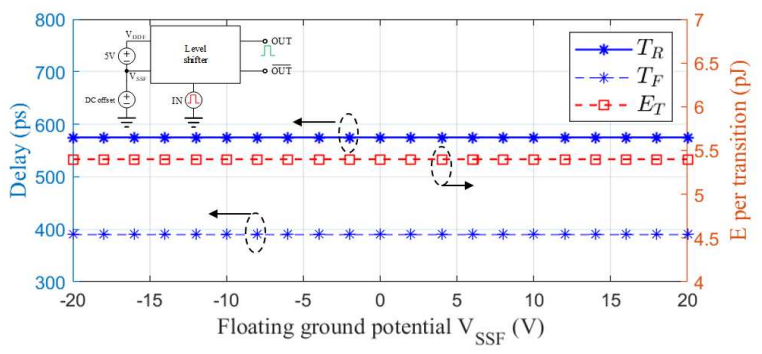

Fig. 4. Simulated delays, energy consumption of the BCC-LS at different floating ground potential.

Fig. 4 shows the pre-layout simulation results of the proposed BCC-LS in terms of rising/falling propagation delays $\left(T_{\mathrm{R}} / T_{\mathrm{F}}\right)$ and energy per transition $\left(E_{\mathrm{T}}\right)$ versus floating ground potential using $180 \mathrm{~nm}$ SOI CMOS process. In this test scheme (shown by the inset), floating supply is set to the fixed values of $5 \mathrm{~V}\left(V_{\mathrm{DDF}}-V_{\mathrm{SSF}}=5 \mathrm{~V}\right)$ then a $\mathrm{DC}$ offset is swept from $-20 \mathrm{~V}$ to $20 \mathrm{~V}$. It can be seen that the propagation delays $T_{\mathrm{R}} / T_{\mathrm{F}}$ are $575 \mathrm{ps} / 390 \mathrm{ps}$, respectively, while the energy per transition $E_{\mathrm{T}}$ is only 5.4 pJ. Especially, the level shifter works whether the potential of $V_{\mathrm{SSF}}$ is negative or positive, which is not the case with AC-LS reported in [9-15]. The insensitive delays versus $V_{\mathrm{SSF}}$ help to achieve delay matching between different floating sides. Furthermore, the energy consumption of the proposed BCC-LS is also a constant and independent of $V_{\text {SSF. The low }}$ delays result from fast level shifter structure using high-bandwidth capacitive couplers and current mirrors, low power consumption and negative $V_{\mathrm{SSF}}$ tolerance is a consequence of using capacitive-coupling technique for the proposed level shifter.

In summary, 3/4 key specifications defined in Section I for a versatile LS can be achieved with BCC-LS: sub-ns delay 
(Ch1), low power consumption (Ch3), and wide negative $V_{\text {SSF }}$ tolerance (Ch4). The low power consumption leads to relaxed requirements for the floating-side power supply, thus making a fully integrated floating supply feasible.

\section{B. High $\mathrm{d} V_{\mathrm{SSF}} / \mathrm{d} t$ capacitive-coupled level shifter}

The proposed BCC-LS is well suited for gate drivers where $V_{\mathrm{SSF}}$ is static such as those driving P-type FETs. However, for deployment in gate drivers driving n-type FETs, the requirement (Ch2) should be satisfied. That is a robust operation under fast $\mathrm{d} V_{\mathrm{SSF}} / \mathrm{d} t$ slewing. With the BCC-LS shown in Fig. 2 , when $V_{\text {SSF }}$ slews up/down with a fast rate, slew-induced common-mode current $I_{\mathrm{cm}}$ appears on both capacitive-coupling branches as the $C_{1}$ and $C_{2}$ need to be charged/discharged. When $V_{\text {SSF }}$ slews up, and assume that node $\mathrm{C}$ is at $V_{\mathrm{SSF}}$ and node $\mathrm{D}$ is at $V_{\mathrm{DDF}}$, slew-induced current $I_{\mathrm{cm}}$ is only copied by the current mirror PM1-PM2 because $V_{\mathrm{ds}, \mathrm{PM} 1}=V_{\mathrm{DDF}}$ while $V_{\mathrm{ds}, \mathrm{PM} 4}=0$. If the induced commonmode current $I_{\mathrm{PM} 2}=N \times I_{\mathrm{cm}}$ is high enough, node $\mathrm{C}$ can be charged up and the output of the LS flips erroneously. When $V_{\mathrm{SSF}}$ slews down, induced $I_{\mathrm{cm}}$ currents are in the reverse direction and discharge to the floating supply $V_{\mathrm{DDF}}$ through the body diodes of PM1 and PM3. This does not cause any effect on the output of the LS. By simulation, the maximum $V_{\mathrm{SSF}} / \mathrm{d} t$ of the basic BCC-LS is only $12 \mathrm{~V} / \mathrm{ns}$. To cope with the high $\mathrm{d} V_{\text {SSF }} / \mathrm{d} t$ requirement, a modified version of the BCC-LS, so-called MCCL-LS is proposed as shown in Fig. 5. The $\mathrm{d} V_{\mathrm{SSF}} \mathrm{d} t$ immunity of the modified LS is enhanced by using two cross-current mirrors (CCMs) [15] and two fast transmission gates (TGs). Brief operation of the MCC-LS is explained below:

+ Differential mode (shifting mode) - Fig. 5(a): At the rising edge of the input IN, a short pulse is generated at the output of the pulse generator $V_{\mathrm{PR}}$. This short pulse pulls down the voltage at node $\mathrm{A} 1$ and generates a current $I_{\mathrm{PM} 1}$ through the diode-connected transistor PM1. While $V_{\mathrm{PF}}$ of the pulse generator is maintained at $V_{\mathrm{DDL}}$, the right half $\left(C_{2}, \mathrm{PM} 3\right.$, PM4, PM6, NM3-NM4) is inactive. The current PM1 is mirrored by PM2-PM5, NM1-NM2, goes through the TGs to charge node D2 and discharge node D1 (in differential mode, TGs are always $\mathrm{ON}$ as either $\mathrm{A} 1$ or $\mathrm{A} 2$ is at $V_{\mathrm{DDF}}$, and either $\mathrm{B} 1$ or $\mathrm{B} 2$ is at $\left.V_{\mathrm{SSF}}\right)$. As node $\mathrm{D} 2$ is charged, source/sink triggering currents $I_{\mathrm{NM} 6} / I_{\mathrm{PM} 10}$ are generated. As a consequence, node F1 is charged via PM10 while node F2 is discharged via NM6; thus, triggering the latch and flip the output OUT to $V_{\mathrm{DDF}}$. It should be noted that since the latch is charged/discharged by PM10/NM6 simultaneously, the latch is triggered faster as compared to BCC-LS shown in Fig. 2. The same working fashion is applied at the falling edge of the input IN to pull OUT down to $V_{\mathrm{SSF}}$.

+ Common mode ( $d V_{\mathrm{SSF}} / d t$ noise cancelling mode) Fig. 5(b): When $V_{\text {SSF }}$ slews fast, induced common mode currents appear on both PM1 and PM3, then copied and cancelled by the first CCM at node $\mathrm{C} 1$ and $\mathrm{C} 2$ (ideally $I_{\mathrm{cm} 1}=$ $\left.I_{\mathrm{cm} 2}\right)$. However, when the induced common mode current is high and due to mismatch (between $C_{1}, C_{2}$; between devices of the first CCM caused by process variations, etc.), the net common mode currents coming out nodes $\mathrm{C} 1$ and $\mathrm{C} 2$, that is $I_{\mathrm{cm}, \mathrm{Cl}, 2}=\left|I_{\mathrm{cm} 1}-I_{\mathrm{cm} 2}\right| \neq 0$. These currents can be further blocked by the transmission gates TG1 and TG2. When $V_{\text {SSF }}$ slews, nodes A1 and A2 are pulled down and nodes B1 and B2 are pulled up. As a consequence, TG1 and TG2 are opened and net common mode currents $I_{\mathrm{cm}, \mathrm{Cl}, 2}$ cannot enter nodes D1 and D2 as the TGs' responses are faster than the current mirrors. As a consequence, the second CCM is not triggered and the latch states are not affected.

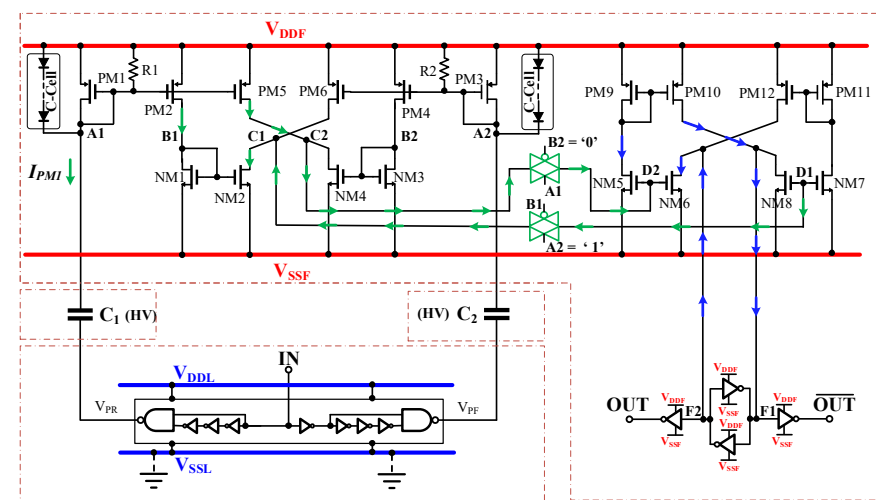

(a)

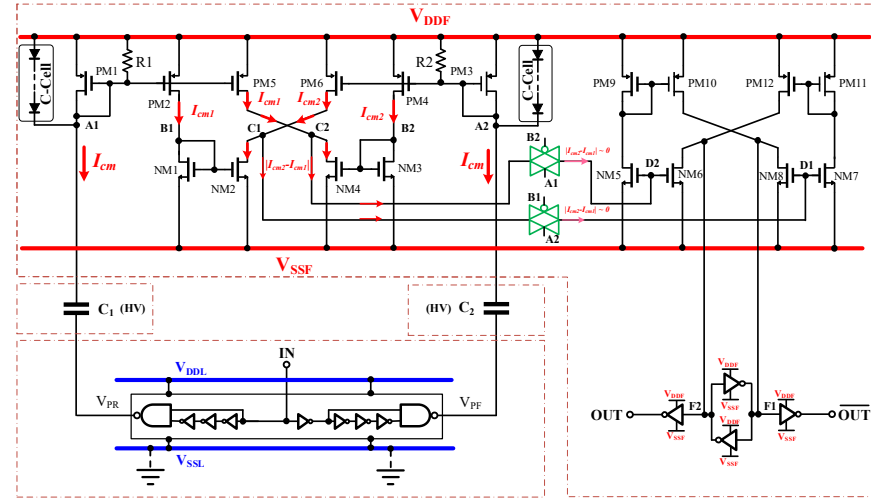

(b)

Fig. 5. Operation of MCC-LS: (a) Differential mode, (b) Common mode (Red dashed boxes are deep $\mathrm{N}$-wells for isolations).

At high $\mathrm{d} V_{\mathrm{SSF}} / \mathrm{d} t$ rate, $I_{\mathrm{cm}}$ is significant and sourcegate/source-drain dropped voltages of PM1/PM4 can be higher than maximum rated values of the utilized standard $5 \mathrm{~V}$ MOSFETs. This can break down low $V_{\mathrm{GS}}$ devices. By using stacked-diode clamping cells (C-cells), $C_{1}$ and $C_{2}$ are charged via these clamping cells and $V_{\mathrm{SG}} / V_{\mathrm{SD}}$ of PM1-PM6 are limited within a safe range. The clamping voltages are designed such that none of the clamping cells is conducted in shifting mode. In summary, as TGs and CCMs are included, $\mathrm{d} V_{\mathrm{SSF}} / \mathrm{d} t$ immunity of the proposed MCC-LS is higher than BCC-LS counterpart with the cost of longer delay

\section{Post-Layout Simulation Results}

The proposed MCC-LS was implemented using $0.18 \mu \mathrm{m}$ HV BCD-on-SOI process supplied by X-FAB. The coupling capacitors $C_{1}$ and $C_{2}$ are $\mathrm{HV}$ devices with a maximum rated voltage of $200 \mathrm{~V}$ and the remaining are standard $5 \mathrm{~V}$ devices. To reduce the induced common-mode currents during fast $\mathrm{d} V_{\mathrm{SSF}} / \mathrm{d} t$ slewing, coupling capacitors $C_{1}$ and $C_{2}$ have a small value of $40 \mathrm{fF}$. Besides, two $300 \mathrm{k} \Omega$ resistors $\mathrm{R} 1$ and $\mathrm{R} 2$ are added between nodes $\mathrm{A} 1$ and $\mathrm{A} 2$ and $V_{\mathrm{DDF}}$ which help to reset these node potentials to $V_{\mathrm{DDF}}$ to be ready for the next pulse, especially for high-frequency operation. The layout and test bench are shown in Fig. 6(a) and 6(b), respectively.

Fig. 7(a) shows the post-layout simulation results of the MCC-LS in terms of delays and energy consumption per transition. The rising/falling delays and energy consumption 
of the MCC-LS are only $686 \mathrm{ps} / 660 \mathrm{ps}$ and $8.1 \mathrm{pJ}$, respectively. This confirms the sub-ns feature of the proposed design. Also, the propagation delays and energy consumption are constant and independent of floating ground $V_{\text {SSF. The }} \mathrm{d} V_{\text {SSF }} / \mathrm{d} t$ immunity of MCC-LS is shown in Fig. 7(b). Even after exposing a slewing rate up to $200 \mathrm{~V} / \mathrm{ns}$ to $V_{\mathrm{SSF}}$ node, the output status follows the input perfectly as the common currents entering node D1 and D2 are wellcancelled. This can be confirmed by low common mode voltage noise at node D1. Therefore, the proposed MCC-LS guarantees a malfunction-free operation up to $200 \mathrm{~V} / \mathrm{ns}$ of $\mathrm{d} V_{\text {SSF }} / \mathrm{d} t$ slew rate. Besides, $V_{\text {PR }}$ of the low side is clamped by body diodes of CMOS devices at the output of the NAND gate of the pulse generator while $V_{\mathrm{SD}}$ of PM1, PM3 of floating side are clamped by body diodes of PM1, PM3 and C-cells; thus $-V_{\mathrm{D}}<V_{\mathrm{DDL}} / V_{\mathrm{DDF}}+V_{\mathrm{D}}$. This verifies that lowvoltage CMOS devices are always operated within safety regions during $V_{\mathrm{SSF}}$ transition.

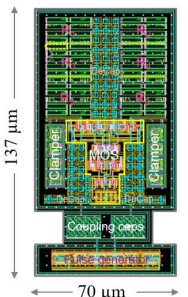

(a)

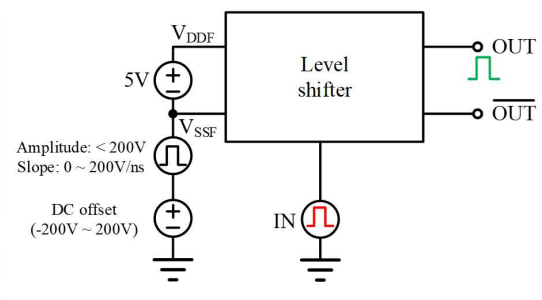

(b)
Fig. 6. (a) Layout of the proposed MCC-LS level shifter, (b) Setup for post simulation of level shifters.

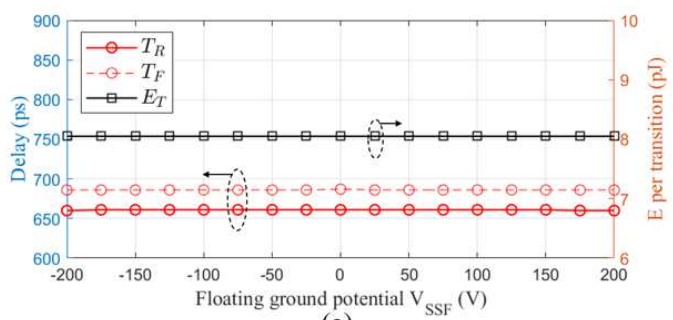

(a)

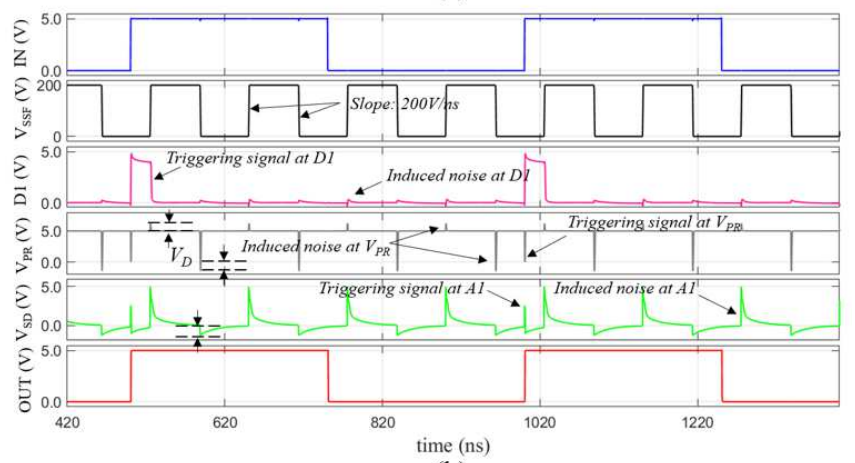

(b)

Fig. 7. Post-sim results of MCC-LS: (a) Propagation delays and energy consumption, (b) $\mathrm{d} V_{\mathrm{SSF}} / \mathrm{d} t$ immunity at $200 \mathrm{~V} / \mathrm{ns},\left(@ \mathrm{f}_{\mathrm{IN}}=20 \mathrm{MHz}, \mathrm{OUT}\right.$ is referenced to $\left.V_{\mathrm{SSF}}\right)$.

Table I summarizes the proposed LS's performance (MCC-LS) along with the prior arts mentioned in Section 2. Among these works targeted for high-voltage gate drivers, the proposed LS can transmit signals with a delay below $700 \mathrm{ps}$, which confirms the sub-ns delay beside the works in [14-15]. The $\mathrm{d} V_{\mathrm{SSF}} / \mathrm{d} t$ immunity of the proposed design is
$200 \mathrm{~V} / \mathrm{ns}$, which is highest and is comparable to [15]. The energy consumption $E_{\mathrm{T}}<8.1 \mathrm{pJ} /$ transition and stays constant regardless of the floating supply $V_{\mathrm{DDF}}$ potential. Only [17] has lower $E_{\mathrm{T}}$, but its delay is higher and $\mathrm{d} V_{\mathrm{SSF}} / \mathrm{d} t$ is much lower. At higher floating supply potential, the benefit of the proposed LS in terms of $E_{\mathrm{T}}$ becomes more significant as compared to AC-LSs in [14-16]. By extrapolation, the LS using active-coupled technique in [16] has $E_{\mathrm{T}}$ of $285 \mathrm{pJ}$ that is higher than $E_{T}$ of this work by a factor of 35 at $200 \mathrm{~V}$ of $V_{\mathrm{DDF}}$. The low energy consumption makes the proposed LS very suitable for the implementation of fully integrated floating supplies for gate drivers. By using capacitive coupling technique, the operation range of the proposed LS is not limited in positive range of $V_{\mathrm{DDF}}$. In particular, the proposed work can shift the signal down to $200 \mathrm{~V}$ of the floating rail.

TABLE I: COMPARISON WITH PREVIOUS WORKS

\begin{tabular}{|c|c|c|c|c|c|c|c|}
\hline REF & $\begin{array}{c}\boldsymbol{V}_{\text {DDF,max }} \\
(\mathrm{V})\end{array}$ & $\begin{array}{c}\boldsymbol{E}_{\mathbf{T}} \\
(\mathrm{pJ})\end{array}$ & $\begin{array}{c}\boldsymbol{V}_{\text {SSF,min }} \\
(\mathrm{V})\end{array}$ & $\begin{array}{c}{ }^{a} \boldsymbol{T}_{\mathbf{d}} \\
(\mathrm{ns})\end{array}$ & $\begin{array}{c}\boldsymbol{d} \text { V/dt } \\
(\mathrm{V} / \mathrm{ns})\end{array}$ & $\begin{array}{c}{ }^{\boldsymbol{b}} \boldsymbol{F} \text { FoM } \\
{ }^{\boldsymbol{c}} \boldsymbol{F} \boldsymbol{F} \boldsymbol{M}^{2}\end{array}$ & $\begin{array}{c}\text { Process } \\
(\mu \mathrm{m})\end{array}$ \\
\hline$[4]$ & 40 & 160 & N/A & 2 & 40 & $0.1 / 64$ & 0.5 \\
\hline$[14]$ & 20 & 27.6 & N/A & 0.37 & 30 & $0.1 / 23$ & 0.18 \\
\hline$[15]$ & 50 & 30.3 & -1.5 & 0.53 & 200 & $0.06 / 55$ & 0.18 \\
\hline$[17]$ & 50 & 4.1 & N/A & 1.45 & 6 & $0.16 / 20$ & 0.18 \\
\hline This work & 200 & 8.1 & -200 & 0.67 & 200 & $0.02 / 4.7$ & 0.18 \\
\hline
\end{tabular}

To evaluate the overall performance of the proposed LS in terms of delay, energy consumption, process dependence, and transfer voltage, two figure of merits (FoMs) are commonly used as benchmarks [14,17]. FoM ${ }^{1}$ takes into consideration of transfer delay, process node length and transfer voltage while $\mathrm{FoM}^{2}$ includes those factors and also reflects power dissipation. The smaller the $\mathrm{FoM}^{1}$, the faster the LS and the smaller and $\mathrm{FoM}^{2}$, the LS is faster and more energy efficient. At $200 \mathrm{~V}$ of floating supply potential $\mathrm{V}_{\mathrm{DDF}}$, the proposed LS has a $\mathrm{FoM}^{1}$ of $0.02 \mathrm{~ns} /(\mu \mathrm{m} \cdot \mathrm{V})$ and an excellent $\mathrm{FoM}^{2}$ of $4.7(\mathrm{pJ} \cdot \mathrm{ns}) /\left(\mu \mathrm{m}^{3} \cdot \mathrm{V}\right)$, thanks to the low delay and low $E_{\mathrm{T}}$. These figures of merits can be considered the lowest as compared to the reported works.

\section{CONCLUSION}

Although many high-speed, high $\mathrm{d} V_{\mathrm{SSF}} / \mathrm{d} t$ immunity level shifters have existed, the limited negative $V_{\text {SSF }}$ tolerance and high energy consumption are the remained drawbacks. This paper has presented a novel capacitive-coupler/current mirror/ latch structure for floating high-voltage gate drivers. The proposed design achieves sub-ns of less than 700 ps of propagation delays and a $\mathrm{d} V_{\mathrm{SSF}} / \mathrm{d} t$ up to $200 \mathrm{~V} / \mathrm{ns}$. Especially, the proposed LS can operate even with negative floating supply and consumes a very low energy consumption of only $8.1 \mathrm{pJ} /$ transition regardless of the value of floating power supply rail. Therefore, the proposed level shifter can be used for gate drivers for both of conventional and emerging DCDC converters. Measurements will be conducted in the future to confirm the performance of the proposed level shifter.

\section{ACKNOWLEDGMENT}

This work was supported in part by the Natural Sciences and Engineering Research Council of Canada (NSERC). The authors would like to thank CMC Microsystems, Canada for $\mathrm{CAD}$ tools and Microsystems Strategic Alliance of Québec (ReSMiQ), Canada for the Postdoctoral Fellow Scholarship. 


\section{REFERENCES}

[1] N. Ly et al., "A High Voltage Multi-Purpose On-the-Fly Reconfigurable Half-Bridge Gate Driver for GaN HEMTs in $0.18-\mu \mathrm{m}$ HV SOI CMOS Technology", in Proc. $18^{\text {th }}$ IEEE Int. New Circuits Syst. Conf. (NEWCAS), pp. 1-4, Aug. 2020.

[2] E. Aklimi, D. Piedra, and K. Tien, "Hybrid CMOS/GaN 40-MHz Maximum 20-V Input DC-DC Multiphase Buck Converter”, IEEE J. Solid-State Circuits, vol. 52 , no. 6, pp. 1618-1627, Mar. 2017.

[3] N. Fichtenbaum, M. Giandalia, S. Sharma, and J. Zhang, "Half-bridge GaN Power IC: Performance and application", IEEE Power Electron. Mag., vol. 4, no. 3, pp. 33-40, Sept. 2017.

[4] Z. Liu, L. Cong, and H. Lee, "Design of On-Chip Gate Drivers with Power-Efficient High-Speed Level Shifting and Dynamic Timing Control for High-Voltage Synchronous Switching Power Converters", IEEE J. Solid-State Circuits, vol. 50, no. 6, pp. 1463-1477, Jun. 2015.

[5] A. Lidow: "eGaN FET Electrical Characteristics", Efficient Power Conversion Corporation (EPC).

[6] A. Abdulslam and P.P. Mercier, "A Passive-Stacked Third-Order Buck Converter with Inherent Input Filtering Achieving $0.7 \mathrm{~W} / \mathrm{mm}^{2}$ Power Density and 94\% Peak Efficiency", IEEE Solid-State Circuits Lett., vol. 2, no. 11, pp. 240-243, Nov. 2019.

[7] X. Ke, Z. Hay, and S. Tian, "Kappa Switching DC-DC Converter with Continuous Input and Output Currents Achieving 86.7\% Input Ripple Suppression and 16dB Peak EMI Reduction", in Proc. Annu. IEEE Appl. Power Electron. Conf. Expo. (APEC), pp. 1315-1319, Jun. 2020.

[8] V. H. Nguyen et al.: “A Novel Minimum-Phase Dual-Inductor Hybrid Boost Converter with PWM Voltage-Mode Controller", in Proc. IEEE Int. Symp. Circuits Syst. (ISCAS), 2021 (accepted).

[9] Y. Moghe, T. Lehmann, and T. Piessens, "Nanosecond delay floating high voltage level shifters in a $0.35 \mu \mathrm{m}$ HV-CMOS technology", IEEE J. Solid-State Circuits, vol. 46, no. 2, pp. 485-497, Feb. 2011.

[10] T. Lehmann, "Design of fast low-power floating high-voltage level shifters", Electron. Lett., vol. 50, no. 3, pp. 202-204, Feb. 2014.

[11] L. Zhong et al, "A Novel Low Delay High-Voltage Level Shifter with Transient Performance Insensitive to Parasitic Capacitance and Transfer Voltage Level", Circuits Syst. Signal Process., vol. 36, pp. 3598-3615, Jan. 2017.

[12] J. Wittmann, T. Rosahl, and B. Wicht, "A $50 \mathrm{~V}$ high-speed level shifter with high DV/DT immunity for multi-MHz DCDC converters", in Proc. 40 th Eur. Solid State Circuits Conf. (ESSCIRC), pp. 151-154, Nov. 2014.

[13] H. A. Yang et al., " $120 \mathrm{~V} / \mathrm{ns}$ Output Slew Rate Enhancement Technique and High Voltage Clamping Circuit in High Integrated Gate Driver for Power GaN FETs", in Proc. $41^{\text {st }}$ European SolidState Circuits Conference (ESSCIRC), pp. 291-292, Nov. 2015.

[14] D. Liu and S. J. Hollis, "Design of 370-ps Delay Floating-Voltage Level Shifters With 30-V/ns Power Supply Slew Tolerance", IEEE Trans. Circuits Syst. II, Exp. Briefs, vol. 63, no. 7, pp. 688-692, Jul. 2016.

[15] D. Liu, S. J. Hollis, and B. H. Stark, "A New Design Technique for Sub-Nanosecond Delay and $200 \mathrm{~V} / \mathrm{ns}$ Power Supply Slew-Tolerant Floating Voltage Level Shifters for GaN SMPS", IEEE Trans. Circuits Syst. I, Reg. Papers, vol. 66, no. 3, pp. 1280-1290, Mar. 2019.

[16] A. Abdelmoaty, M. Al-Shyoukh, and A. Fayed, "A High-Voltage Level Shifter with Sub-Nano-Second Propagation Delay for Switching Power Converters"," in Proc. Annu. IEEE Appl. Power Electron. Conf. Expo., pp. 2437-2440, May 2016.

[17] D. Lutz, A. Seidel, and B. Wicht, "A 50V, 1.45ns, 4.1pJ High-Speed Low-Power Level Shifter for High-Voltage DCDC Converters", in Proc. $44^{\text {th }}$ European Solid-State Circuits Conference (ESSCIRC), pp. 126-127, Oct. 2018.

[18] L. Peng et al, "A Simple Low Cost Monolithic Transformer for HighVoltage Gate Driver Applications", IEEE Electron Device Lett., vol. 35 , no. 1 , pp. 108-110, Jan. 2014 